\title{
Creation of regular arrays of faceted AIN nanostructures via a combined top-down, bottom-up approach
}

\author{
R. Armstrong*,1, P-M. Coulon ${ }^{1}$, P. Bozinkas ${ }^{2}$, R. Martin ${ }^{2}$ and P. Shields ${ }^{1}$ \\ ${ }^{1}$ Department of Electronic and Electrical Engineering, University of Bath, Claverton down, Bath, United Kingdom, BA2 7AY \\ ${ }^{2}$ Department of Physics, SUPA, University of Strathclyde, John Anderson Building, 107 Rottenrow, United Kingdom, Glasgow, G4 ONG
}

\section{ARTICLE INFO}

Keywords:

B1. Nitrides

B2. Semiconducting aluminum compounds

A1. Surface structure

A3. Metalorganic chemical vapor deposition

A3. Metalorganic vapor phase epitaxy A1. Nanostructures

\begin{abstract}
A B S T R AC T
The realisation of spatially-determined, uniform arrays of faceted aluminium nitride (AlN) nanostructures has had limited exploration, largely due to the fact that selective area growth of AlN via MOVPE (Metal Organic Vapour Phase Epitaxy) has not been realised. Instead, this paper reports the use of a combined top-down, bottom-up approach to realise well-faceted, highly-uniform, periodic nanotextured AlN surfaces. MOVPE regrowth is performed upon dry-etched AlN nanorods and nanoholes, and we present a study into the effect of the growth conditions on the resulting faceting and morphology. Specifically, growth temperature, V/III ratio and growth time are investigated and analysed via scanning-electron and atomic-force microscopy. The V/III ratio was found to influence the nanostructure morphology most whilst the growth temperature was found to have much less of an impact within the temperature range studied. Experiments with a longer growth time are performed to create nanostructures for potential use in applications, such as for AlGaN-based quantum-well or quantum-dot emitters.
\end{abstract}

\section{Introduction}

Aluminium Nitride (AlN), Gallium Nitride (GaN) and their alloys are wide- [1] and direct-band-gap semiconductors when in the stable, wurtzite, crystal polytype [2]. The band gaps for $\mathrm{AlN}$ and $\mathrm{GaN}$ are $\sim 6.2 \mathrm{eV}$ and $\sim 3.4 \mathrm{eV}$ respectively [3]. The large band-gap range of AlN and GaN means that alloys of these two materials, with high $\mathrm{Al}$ content, are suitable for UVC devices across the range of 200-280 nm. However, planar AlN-based UVC devices suffer from low quantum efficiency and output power [4]. This is partially due to large amounts of non-radiative recombination in these devices. The main cause for non-radiative recombination in AIN and AlGaN based UV LEDs are the high levels of threading dislocation densities and point defects. This leads to an inevitable reduction in the internal and external quantum efficiencies [5]. High device operation voltages are also necessary [6] and the polarisation coefficient of emission also acts as a bottleneck for high-performance UVC applications [7]. The polarisation coefficient is important as TM polarised light is predominantly emitted from high-Al-contentAlGaN- and AlN-based planar LEDs. Light of this polarisation is very difficult to extract from c-plane AlN or highAl-content-AlGaN material [8] [9]. This, therefore, leads to heavily-reduced light extraction efficiencies [4].

3D structures can, however, be used to overcome these limitations to some extent. Nanopatterning of sapphire/AlN templates has been shown to be effective at lowering threading dislocation densities [10] [11] [12]. 3D nanostructures also allow for stress relaxation, increased active volume [13] and higher extraction efficiencies [14]. In the case of higher extraction efficiencies, utilising nanostructures has been found the be very effective in allowing the escape of TM-polarised

\footnotetext{
을 ra760abath.ac.uk (R. Armstrong) ORCID(s):
}

photons [15] due to the presence of non- and semi-polar planes from which TM polarised light can escape with greater ease.

AlN nanostructures can be used to house more exotic light emitters such as quantum dots. For this, ideally the nanostructures would comprise highly uniform and ideally spatially-predetermined arrays, to aid electrical injection. Single photon emission (SPE) from III-N site-controlled quantum dots has been demonstrated to operate above room temperature [16]. Arrays of quantum dots which offer spatial, shape and size determination leading to identical properties are particularly suitable for quantum information processing (QIP) applications [17, 18, 16]. Previously, SPE from GaN quantum dots at [19] and above room temperature [16] was achieved using $\mathrm{GaN}$ nanorods created via selective area growth (SAG). This was followed by growth of an $\mathrm{Al}_{x} \mathrm{Ga}_{1-x} \mathrm{~N}$ shell $(x=0.8), \mathrm{GaN}$ quantum dot growth and finally a capping layer [20]. Ideally, one would desire a pure AlN barrier layer to increase the band offsets, resulting in better confinement, in order to realise even higher temperature SPE. Additionally, the use of a purely AlN core would avoid photon reabsorption problems associated with a $\mathrm{GaN}$ core. However, current methods to achieve AlN nanostructures are not welldeveloped and, using pure AlN as a shell layer on SAG GaN nanostructures without considering the nanostructure core, is insufficient [20]. Therefore, another route for the creation of uniform, well-faceted, high-quality arrays of AlN nanostructures for both optoelectronic UVC applications and as sites for quantum dots is necessary.

AlN nanostructures have been fabricated via a number of different routes; AlN nanowires [21], via arc discharge [22], vapour-liquid-solid [23] and vapour-solid [24] techniques, in addition to MOVPE growth utilising catalyst droplets [25]. However, achieving dimensionally-uniform structures is very challenging [21] and no spatially-predetermined arrays have 
been realised. One of the most widely-employed methods is the growth of self-organised AlN nanorods via radio-frequency plasma-assisted molecular beam epitaxy [6]. Whilst these rods are somewhat uniform and vertically aligned [6] they are not homogeneous in their shape and they nucleate randomly. As has been discovered with GaN self-assembled nanorods, these structures will exhibit non-uniform properties and may hinder device processing [26]. Specifically, the non-uniform dimensions of the rods will lead, upon growth of active regions, to in-homogeneous optoelectronic properties of the rod arrays. Circumventing these problems would obviously be desirable in device applications.

Recently, a catalyst- and lithography-free method for the fabrication of AlN nanorods by polarity selective epitaxy and etching has also been demonstrated [14]. Here, an AlN layer of mixed polarity is grown and wet etching is used to selectively etch away N-polar regions of material [6]. Multiple quantum wells were then grown on the resultant nanorods in a core-shell architecture. However, the rods had random spatial locations that led to non-uniform regrowth of the active regions.

Selective area growth (SAG) is an effective method for the creation of faceted, highly-uniform, spatially-predetermined arrays of nanostructures. This has been widely utilised for the growth of GaN nanostructures such as nanopyramids [27] and nanorods [28]. It has also been demonstrated with InN; again the growth of nanopyramids [29] and nanorods [30] have been achieved. However, with AlN, no demonstration of SAG, via MOVPE, has been demonstrated thus far. This is because aluminium $(\mathrm{Al})$ adatoms have a very short diffusion length and a large sticking coefficient [31]. This means that they cannot diffuse effectively across a dielectric mask to any more favourable, native, bonding sites. This results in parasitic growth upon the mask itself instead of the formation of AlN nanostructures within the mask apertures.

The combined top-down, bottom-up approach is a promising approach to achieve complex nanostructures [10] [11] [32] [33]. This process involves the nanopatterning and topdown etching of a material (such as an initial planar AlN template) to create nanostructures. This is followed by bottomup regrowth leading to well-faceted nanostructures. Any surface damage introduced from dry etching is buried during regrowth or removed by wet etching to limit its impact on device performance. This technique has been used to create arrays of $\mathrm{GaN} / \mathrm{InGaN} / \mathrm{GaN}$ core-shell structures [34] [32] and AlN/AlGaN/AlN structures [35] [33]. However, this technique is predominantly used for regrowth onto nanorods, with other structures largely unexplored.

The nature of the faceting that occurs during regrowth on structured substrates (as shown later in this paper) allows for an enhancement of the resolution of conventional lithographic processes in order to create small nucleation regions for the growth of quantum dots. Such regions are typically much smaller than the resolution of large-area lithography techniques that are widely available to researchers. Moreover, access to well-organised semi- and non-polar facets opens up avenues to reduce the impact of the quantum-confined
Figure 1: SEM images of the four nanostructures created. Shown are the deep etched nanorods (a) and nanoholes (c) ( $\sim 1 \mu \mathrm{m}$ deep) and shallow etched nanorods (b) and nanoholes (d) $(\sim 100 \mathrm{~nm}$ deep). The insets show the plan-view SEM images of the rods and the cross-section SEM images of the holes.
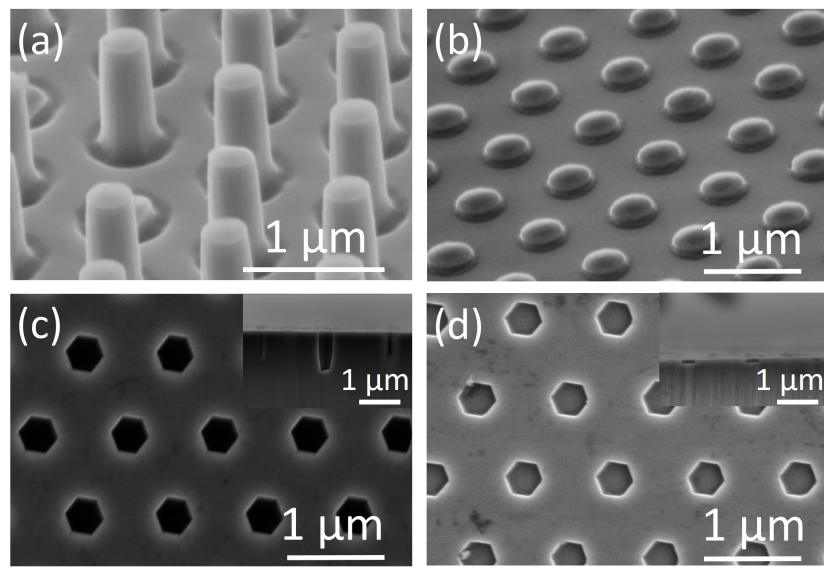

Stark effect (QCSE) present in active regions suitable for UVC emission applications in planar devices [35].

In this work, we present the fabrication of AlN nanorod and nanohole structures, followed by a study and analysis of the effect of different MOVPE regrowth conditions. The growth dynamics of planes of different orientations is presented and discussed for both nanorods and nanoholes. We also discuss the results with regards to suitability for the applications mentioned.

\section{Materials \& Methods}

Commercially-sourced, planar, $\sim 4.5 \mu \mathrm{m}$ thick, AlN templates (Suzhou Nanowin Science and Technology Co. LTD, Suzhou, China) were used to fabricate nanorod and hole structures at two different etch depths. The fabrication process involved the use of Displacement Talbot Lithography (a form of photolithography) [36] [37] in order to pattern photoresist for either the deposition or patterning of a hard etch mask onto the AlN templates. This hard mask was then used during a ICP (inductively coupled plasma) dry etch to create hole and rod structures with depths of $1 \mu \mathrm{m}$ and $\sim 100 \mathrm{~nm}$. The two different etch depths were chosen to study the impact of the aspect ratio of the structures on the regrowth dynamics taking place.

Figure 1 shows the nanostructured templates after the dry etching and removal of the hard mask. This Figure presents a number of interesting observations. As one can see in (a) and (b) the rod samples are relatively circular with slight hexagonal faceting exhibiting 'edge-to-edge' morphology observed in the deep rod sample (as can be seen from the inset in (a)). The hole samples ((c), (d)) by contrast, exhibit very clear and distinctive hexagonal faceting with 'point-to-point' morphology. By comparing the geometry with the flat of the sapphire wafer, the sidewall facets can be determined to be 
Table 1

Details of the growth experiments with the changes highlighted in red. For all growth experiments the TMAl flow was $20 \mathrm{sccm}$, the pressure was $20 \mathrm{mbar}$ and the carrier gas was $\mathrm{H}_{2}$.

\begin{tabular}{|c|c|c|c|c|c|}
\hline Growth run & Set temperature ${ }^{\circ} \mathrm{C}$ & $\mathrm{NH}_{3}$ Flow $(\mathrm{sccm})$ & $\mathrm{V} / \mathrm{III}$ ratio & Growth time $(\mathrm{min})$ & Corresponding figures \\
\hline 1 & 900 & 1000 & $\sim 3800$ & 60 & $2(\mathrm{a}),(\mathrm{d}),(\mathrm{g}),(\mathrm{j})$ \\
\hline 2 & 950 & 1000 & $\sim 3800$ & 60 & $2(\mathrm{~b}),(\mathrm{e}),(\mathrm{h}),(\mathrm{k})$ \\
\hline 3 & $980 / 1005^{1}$ & 1000 & $\sim 3800$ & 60 & $2(\mathrm{c}),(\mathrm{f}),(\mathrm{i}),(\mathrm{l})$ \\
\hline 4 & 950 & 20 & $\sim 74$ & 60 & $3(\mathrm{a}),(\mathrm{e}),(\mathrm{i}),(\mathrm{m})$ \\
\hline 2 & 950 & 1000 & $\sim 3800$ & 60 & $3(\mathrm{~b}),(\mathrm{f}),(\mathrm{j}),(\mathrm{n})$ \\
\hline 5 & 950 & 2000 & $\sim 7600$ & 60 & $3(\mathrm{c}),(\mathrm{g}),(\mathrm{k}),(\mathrm{o})$ \\
\hline 6 & 950 & 5000 & $\sim 19000$ & 60 & $3(\mathrm{~d}),(\mathrm{h}),(\mathrm{l}),(\mathrm{p})$ \\
\hline 5 & 950 & 2000 & $\sim 7600$ & 60 & $4(\mathrm{a}),(\mathrm{e}),(\mathrm{i}),(\mathrm{m})$ \\
\hline 7 & 950 & 2000 & $\sim 7600$ & 120 & $4(\mathrm{~b}),(\mathrm{f}),(\mathrm{j}),(\mathrm{n})$ \\
\hline 8 & 950 & 2000 & $\sim 7600$ & 150 & $4(\mathrm{c}),(\mathrm{g}),(\mathrm{k}),(\mathrm{o})$ \\
\hline 9 & 950 & 2000 & $\sim 7600$ & 180 & $4(\mathrm{~d}),(\mathrm{h}),(\mathrm{l}),(\mathrm{p})$ \\
\hline
\end{tabular}

${ }^{1}$ In run 3 the two set temperatures correspond to that of two different growth runs for the deep and shallow structures. These temperatures were measured to be the same true temperature measured via emissivity-corrected pyrometry.

$m$-planes $(\{10 \overline{1} 0\})$. Also of note, is the clear difference in etch depth as shown by the insets of (c) and (d).

A series of regrowth experiments were performed (see Table 1). The effect of the growth temperature (runs 1-3), the V/III ratio (runs 4, 2, 5, 6) and a growth time study (runs $5,7,8,9)$ was performed. The growth set temperature was the temperature set via the growth reactor and this was measured to have an offset of up to $\sim 100{ }^{\circ} \mathrm{C}$ less that that of the true temperature measured via an emissivity-corrected pyrometer. The regrown AlN nanostructures were then characterised via SEM and AFM. More details of the fabrication, regrowth and characterisation procedures are given at the end of this paper in the methods section.

\section{Results \& Discussion}

The wurtzite crystal structure, being the most thermodynamically favourable crystal polytype for AlN, leads to the possible presence of a number of planes which can be categorised as polar (normal to the plane parallel to the $c$ axis), non-polar (normal perpendicular to the $c$-axis) and semi-polar upon regrowth of nanostructures. The resulting shape of the structures depends on the speed of the different planes and the topography of the template. For convex structures, such as the nanorods, the slowest growing facets determine the resulting geometry of the structure, whereas for concave structures, such as a shrinking void, the fastest growing planes determine the final shape [38].

Given that the growth speeds of the different planes will alter depending on the growth conditions, we now present the results of varying the growth temperature, V/III ratio and growth time.

\subsection{The effect of growth temperature}

Figure 2 presents SEM images of the growth on the four different templates as a function of the growth temperature whilst keeping all other growth conditions the same. The growth conditions are shown in runs 1-3 of Table 1.
The deep rod samples in Figure 2 (a) - (c) have a 'pyramidon-rod' structure with neighbouring structures being 'pointto-point'. The side facets are $m$-plane as a result of this being the slowest-growing non-polar plane. However, the sidewall facets are limited and the corners between them are not fully formed. With increasing growth temperature, the hexagonal shape of the rods is more well defined with sharper corners. This is likely due to the increased adatom diffusion at higher temperatures. The top $c$-plane truncation is small at all growth temperatures due to the $c$-plane being fast growing in all cases compared to that of the semi-polar planes. Finally, the sidewall facets are almost vertical at the base of the rod, with no presence of semi-polar facets, and tapers slightly outwards as they meet the base of the pyramid.

All the shallow rod samples (Figures 2 (d) - (f)) have large top $c$-planes surrounded by semi-polar planes resulting in an 'edge-to-edge' morphology. Non-polar planes are not present due to the small aspect ratio resulting in either a rapid extinction or no initial formation of the non-polar planes. As the growth temperature increases the features appear less hexagonal, indicative of greater competition between different semi-polar facets. In addition, the semi-polar plane relative to the $c$-plane growth rate increases resulting in rods with large diameters. Therefore, no nanopyramids are formed. Many defects are observed that reduce in number as the growth temperature increases due to the increased adatom diffusion at higher temperatures that allows the defects to be planarised. These defects might have been initiated by the dry-etching step or from pre-existing dislocation pits.

Figure 2 shows the effect of temperature on the deep (g) - (i) and the shallow (j) - (l) hole samples. Both sets show improving regularity of the features with increasing temperature. At the lower temperatures, for the deep hole templates in Figure $2(\mathrm{~g})-(\mathrm{h})$, irregular facets are formed on the semipolar planes, whereas for the shallow hole templates, an unusual morphology is observed for the lowest temperature in Figure 2 (j) due to semi-polar facet competition, and a 'star 
Figure 2: SEM images corresponding to the four templates grown at different temperatures. The green hexagons in (g)- $(\mathrm{l})$ represent the original morphology of the dry etched structure superimposed on the regrown holes. The red arrows in (k) indicate some of the macrosteps that are discussed in the text.
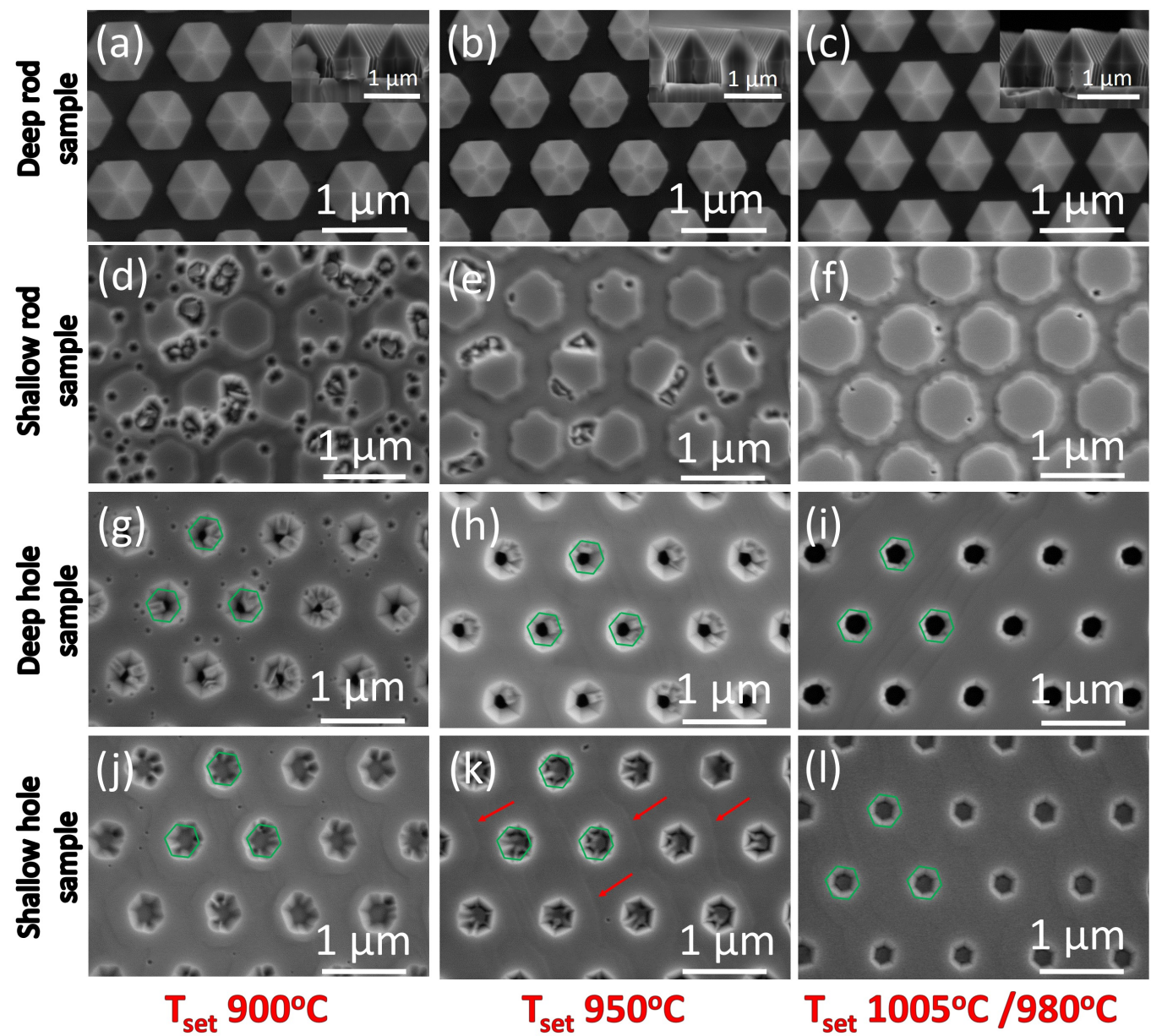

like' growth for a mid-range temperature in Figure $2(\mathrm{k})$ as a result of growth upon the central c-plane of the hole.

If one looks at the outer contrast of each hole, showing the intersections between the semi-polar sidewalls and the top-c-plane, it can be seen that all of the deep hole samples and all of the shallow hole samples, except for the one at the lowest temperature, exhibit edge-to-edge morphology. This indicates the presence of semi-polar $a$-planes $(\{11 \overline{2} i\})$, in stark contrast to the $m$-plane faceted holes after etching. Indeed, the unusual morphologies at low temperature might result from the transition between the $m$-plane facets $(\{10 \overline{1} 0\})$ of the dry etch templates to the semi-polar $a$-planes after regrowth. In contrast, if one looks at the inner contrast of each deep hole sample, the central voids are larger at a higher growth temperature and less hexagonal, indicating less growth and a less dominant non-polar plane.

Macrostep features are visible on the c-plane which appear to bend around both hole samples and appear to bend less at higher growth temperatures.

\subsection{The effect of V/III ratio}

Figure 3 depicts the impact of the V/III ratio on the regrowth of AlN for all four types of templates. Four different V/III ratios were chosen ranging from a very low value of $\sim 74$ to a very high value of $\sim 19000$ (see Table 1, growth runs $4,2,5,6)$.

Figure 3 (a) shows that at very low V/III ratio no smooth non-polar planes are formed on the deep rods. It is likely that rapid extinction of the non-polar planes gives rise to this very undulating, rough sidewall rather than a smooth non-polar facet. The presence of a large top $c$-plane is due to the growth of the $c$-plane being considerably slower than the semi-polar planes with these conditions.

As the V/III ratio increases, the non-polar planes form and intersect each other more closely, with the height of the non-polar planes increasing 3 (b) - (d). The tendency for higher non-polar sidewalls and sharper intersections suggests that the relative speed of the $m$-plane growth decreases as the V/III ratio increases; thus extinguishing any residual $a$ planes. The diameter of the top $c$-plane also increases from 3 (b) - (d) indicating that the relative speed of the semi-polar planes increases. For the highest V/III ratio, the inset in Fig- 
Figure 3: SEM images of the four structures grown with different $V / I I I$ ratios. The green hexagons in $(j)-(I)$ and $(n)-(p)$ represent the original morphology of the dry etched structure superimposed on the regrown holes.

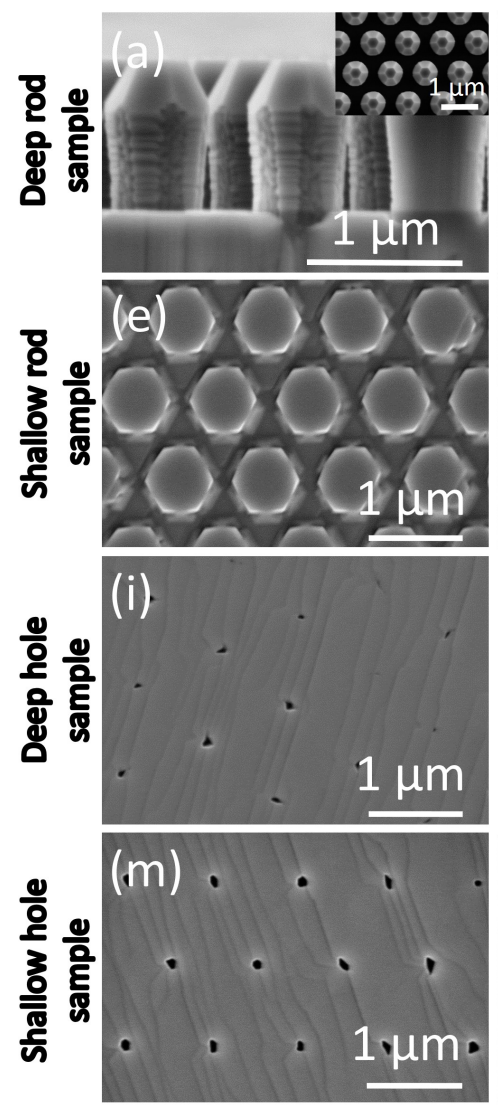

V/III 74
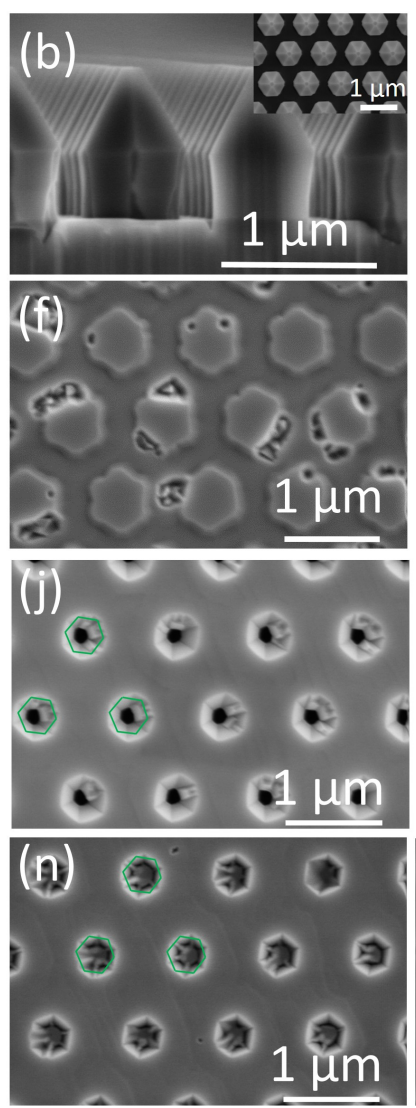

V/III $\sim 3800$
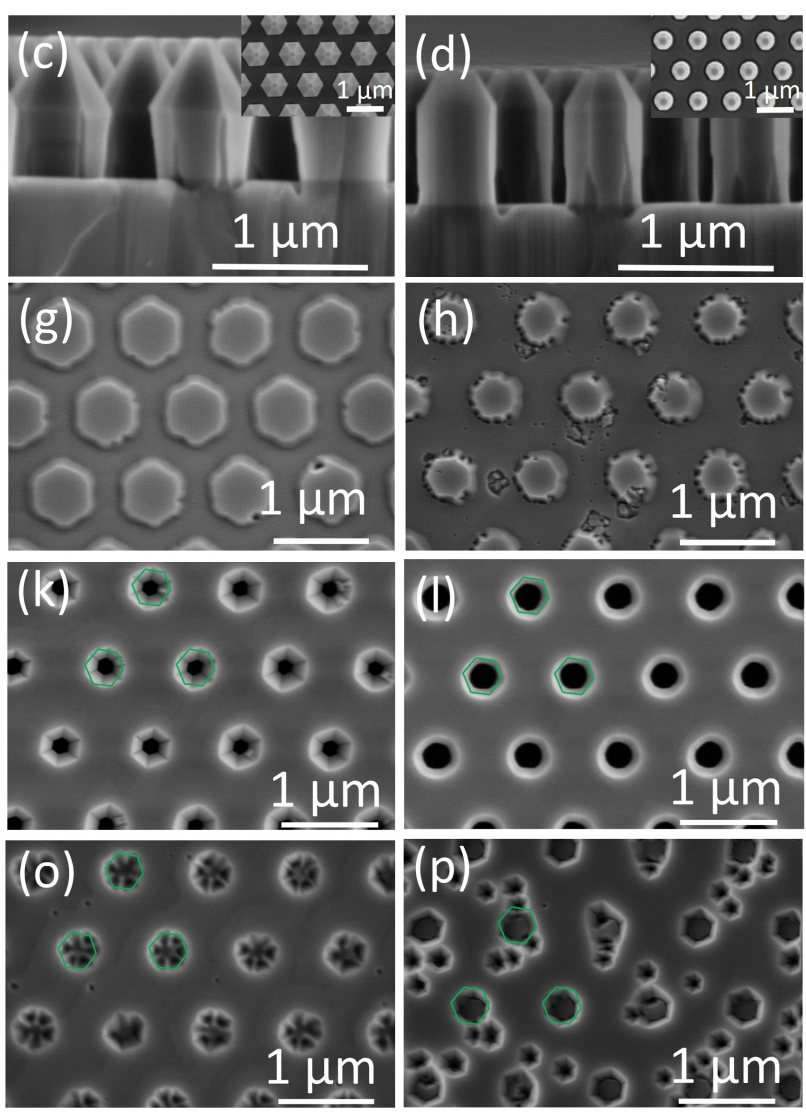

V/III $\sim 7600$

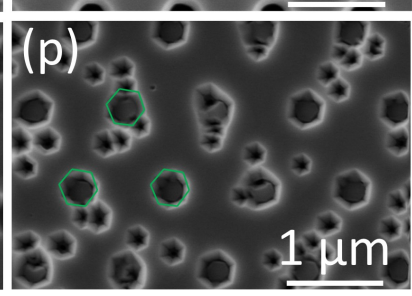

V/III 19000 ure 3 (d) shows a much smaller increase in rod diameter due to a significantly reduced non-polar growth rate.

By examining the height of the nanorods after growth, it can be seen that the nanorods reduce in height with increasing V/III ratio. As the nanorod height is found to be equal or lower than the initial $1 \mu \mathrm{m}$ tall dry etched nanorod, the $c$ plane growth rate at the bottom $c$-plane, in between the rods, is equal or higher than the one on the top nanorod $c$-plane. The reason for this is unclear, but the effect of the slower top-nanorod $c$-plane growth rate is to limit the reduction in lateral size of the $c$-plane facet on top of the nanorod for the high V/III ratios.

Growth with a very low V/III ratio on shallow rods (figure 3 (e)) gives rise to well-defined 'point-to-point' structures in contrast to all other samples ((f) - (h)). No inclined semi-polar planes are observed, with the structure being defined by slow-growing $m$-planes; slower than the non-polar $a$-plane or other semi-polar planes. The lack of semi-polar planes at V/III $\sim 74$ is also observed in the nanohole samples.

As the V/III ratio increases (figure 3 (f)-(g)), the shallow rods change from being 'point-to-point' to 'edge-to-edge' with the sidewalls switching from non-polar $m$-planes to semipolar $a$-planes. At very high V/III ratio ( 19000) (figure 3 (h)) the rods appear multifaceted with a mixture of 'point-topoint' and 'edge-to-edge' morphology. Given that, like for the deep rods, they also exhibit a smaller diameter, it is likely that the lack of growth has prevented the structure finding a more equilibrium and uniform shape.

Turning to the hole samples, no semi-polar planes are formed for either shallow or deep holes at the lowest V/III ratio (figure 3 (i) and (m)), leading to rapid planarisation of the hole array and the likely generation of subsurface voids. For higher V/III ratios, the semi-polar $a$-planes appear along with either other irregular facets in the deep hole samples that reduce when the V/III ratio increases from $\sim 3800$ to $\sim 7800$, or star-like features in the shallow hole samples originating from the competition with the central $c$-plane of the hole.

At a very high V/III ratio of $\sim 19000$ the deep holes (figure 3 (1)) are very circular due to facet competition and an overall lack of growth; also observed in the shallow hole sample (figure $3(\mathrm{p})$ ).

Macrosteps dominate the images of the hole samples at a very low V/III ratio with the height of the steps decreasing with increasing V/III ratio with none being visible in Figure 3 (1) and (p). This is due to the increase in nitrogen atoms on the surface reducing Al-adatom diffusion resulting in shal- 
lower steps [39]. Furthermore, there is minimal perturbation of the macrosteps around the remaining holes in both Figure 3 (i) and (m). This differs from all other growth conditions, both in Figure 2 and 3, where the macrosteps bend around the holes, likely due to the semi-polar planes that are also present.

In an attempt to identify the specific semi-polar planes in the hole samples, their angles were extracted from the AFM scans as shown and detailed in the supplementary information. It was found that, for the deep hole samples, at V/III ratios of $\sim 3800$ and $\sim 7600$ and the shallow holes at V/III ratios of $\sim 3800$ and $\sim 19000$, the planes were oriented around $58-62^{\circ}$ to the horizontal. Combining this with the knowledge of the orientation of the sample and the 'edgeto-edge' morphology leads to the likelihood of these being $\{11 \overline{2} 2\}$ facets. At a V/III ratio of $\sim 19000$ for the deep holes and $\sim 7600$ for the shallow holes the facets are not well defined.

To summarise the results from these growth studies with respect to the original aim of creating suitable sites for quantum dots, it was found that the deep nanorod sample with a V/III ratio of $\sim 7600$ provided a well-faceted structure along with a small $c$-plane truncation. Moreover, the deep hole sample under the same growth conditions was also the most promising, with minimal undesirable faceting on the semipolar planes. Therefore, these conditions were chosen as a starting point for studies of the growth time.

\subsection{The effect of the increase in growth time}

Figure 4 displays the effect of increasing the growth time for the four types of templates (see Table 1 runs $5,7,8,9$ ). For the deep rod samples in Figures 4 (a) - (d)), increasingly sharp apices at the peaks of the pyramidal regions are formed with increasing growth time. The tilted SEM image in Figure 5 from a system capable of higher resolution shows the sharpness more strikingly along with the considerable lateral expansion of the deep rod sample under prolonged growth time that results in rod coalescence.

An overhang is observed at the base of the nanopyramid that is much more pronounced in figures 4 (c) and (d), after the apex is fully formed. The overhang arises due to preferential incorporation of material around this convex region of the rod (see section 3.4). More material arrives at the pyramidal section compared to the sidewalls of the rod, but the slow growth rate of the semi-polar planes leads to adatom diffusion to neighbouring planes. Once the $c$-plane is extinguished, the overhang region is the only sink region for these adatoms; hence the rapid increase in its size. This effect is compounded by restricted gas diffusion to the base of the rod caused by the presence of the large semi-polar planes.

The shallow rods show increasing planarisation and one would expect that further prolonged growth would lead to coalescence. The rods exhibit an 'edge-to-edge' morphology with increased growth time, indicative of much faster semi-polar plane growth compared to that of $c$-plane [11]. The fast growing semi-polar $a$-plane contrasts with the very slow growing semi-polar $m$-planes forming the nanopyra- mids for the deep rod structures, and is due to them having a concave rather than a convex growth nature. This results in the fastest growing planes dominating the rod sidewall morphology, leading to a lateral expansion of the rods.

For the deep hole samples, the semi-polar facets increase in size as the growth time is increased (see Figures 4 (i) - (1)) due to its slow growth rate compared with that of the c-plane. The nanopyrmaidal pits in Figure 4 (j) are close to coalescing with only one family of 6 facets present. With further growth, the holes are found to never fully coalesce (see insets of Figure 4 (j) - (l)) and a second family of facets are visible that compete with each other once this local equilibrium shape has been reached (Figure 4 (1)). Irregular facets also appear for the highest growth times (Figure 4 (l)).

Figure $4(\mathrm{~m})$ - (p) shows the effect of prolonged growth on the shallow holes. After 120 and 150 min the structures themselves appear to shrink in size. At 120 min growth they have well-defined 'edge-to-edge' facets with minimal competition. However, after a growth time of $150-180 \mathrm{~min}$, ridges (or joins) appear to form within the holes leading to an irregular morphology without a well defined hexagonal shape and sometimes elongated in seemingly arbitrary directions. The latter is due to anisotropic planarisation of the hole, on the way to full coalescence and planarisation.

\subsection{Discussion of Growth Mechanisms}

This section describe the mechanism for how the morphology of each template influences the form of the final structure independently of the growth parameters. Whilst this morphology is the major determining factor, the growth parameters primarily influence the final structure through modifications of the relative growth rates of different planes.

Figure 6 shows schematics of the different samples highlighting various critical points and whether they have a convex or concave shape. Note that this shape determines whether the equilibrium morphology is determined by the slowestor fastest-growing crystal facets. However, points on the surface can be characterised by their shape in two dimensions, leading to situations where it is convex along one line (blue arrow in figure 6), and concave along its perpendicular (yellow arrow in figure 6), corresponding to a saddle point. This is in addition to the cases where it is convex or concave in both directions, referred to as double-convex or doubleconcave in the following descriptions, or indeed where it is flat in one or two dimensions. This convex-concave or double convex (concave) morphological nature then determines the shape of towards which the structure grows, as presented in the model described in [40]. Although in [40] this is considered for GaN SAG structures this can also be applied to our AlN top-down bottom-up nanostructures.

In the deep rod sample (Figure 6a) a double-convex base of a semi-polar pyramid forms in the upper region of the rod. Due to the reduced number of bonding sites being available at this corner it is more costly for adatoms to bind here. Thus this region is slower growing than other regions and leads to the slow-growing semi-polar planes dominating the rod structure after prolonged growth times. This manifests 
Figure 4: SEM images corresponding to the growth on the four structures for different regrowth times. The green hexagons in (i)-(p) represent the original morphology of the dry etched structure superimposed on the regrown holes.
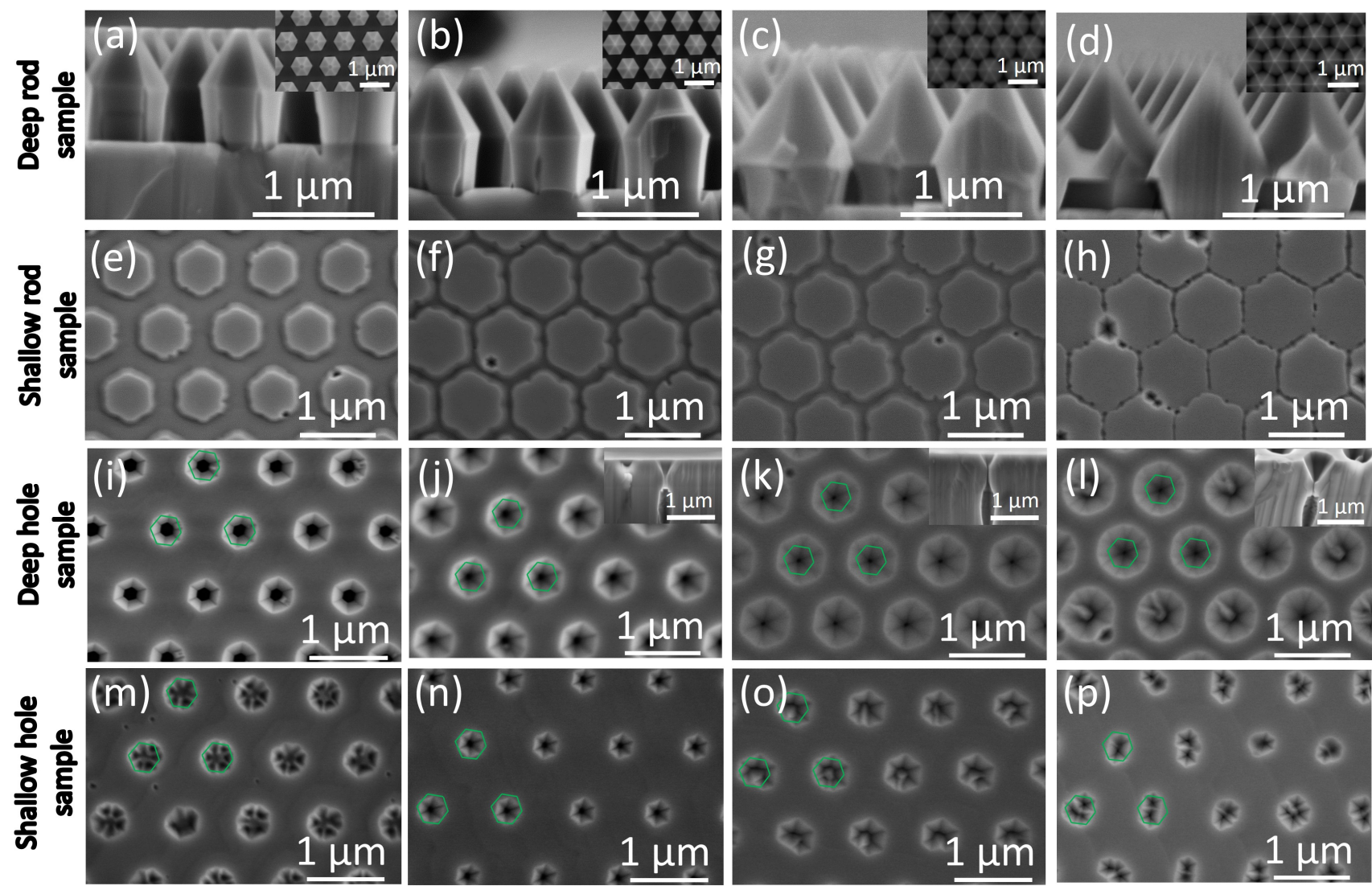

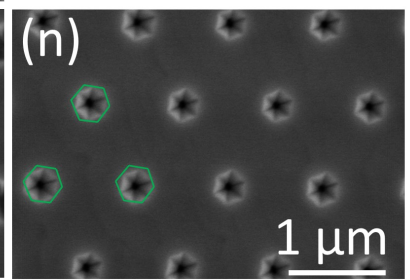

Growth time 120 minutes

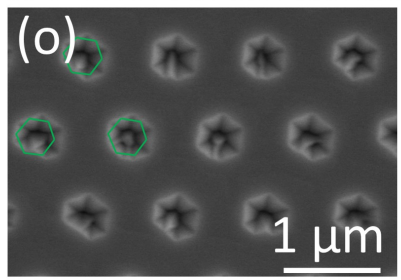

Growth time 150 minutes

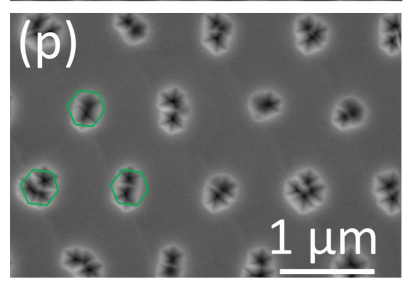

Growth time 180 minutes
Figure 5: High resolution tilted SEM image of the sample from growth run 9.

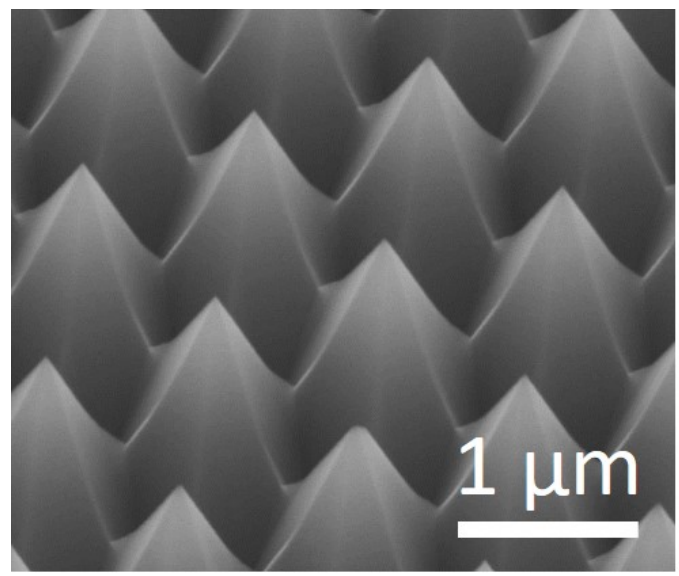

itself with a visible overhang and an increase of the size of the semi-polar planes relative to other plane geometries, also leading to the top c-plane being extinguished.

In the shallow rod sample (Figure 6b), the base of the rods has a concave morphology in one direction so that there are more bonding sites available than in other regions. This results in a more rapid growth of the semi-polar planes leading to no formation of any non-polar planes and the fastgrowing semi-polar planes dictating the final morphology of the structure. In the absence of the non-polar planes, prolonged growth results in lateral expansion of the rods. This also results in an expansion of the top c-plane as it is slower growing relative to the semi-polar planes resulting in an almost coalesced layer after the longest growth time, despite the top of the rods having double convex morphology.

Also of note is that these structures have 'wavy' edges. This is likely due to the impact of the irregular dry etch trench on this critical convex-concave region which is driving the speed of the semi-polar growth.

In the deep hole samples (Figure 6c) the intersections of the planes are mostly convex in nature, with concave regions between families of semi-polar planes and between families of non-polar planes. The concave nature leads to the formation of the faster-growing semi-polar and non-polar $a$-plane facets. However, the growth rate of these semi-polar planes is still slow enough for the semi-polar planes to continue to 
Figure 6: Schematic representation of the convex and concave nature of all four structures. The images on the left are before regrowth and the images on the right after regrowth. The deep rod, shallow rod, deep hole and shallow hole structures are shown in (a), (b), (c) and (d) respectively. Blue arrows indicate convex morphology whereas yellow arrows indicate concave morphology.

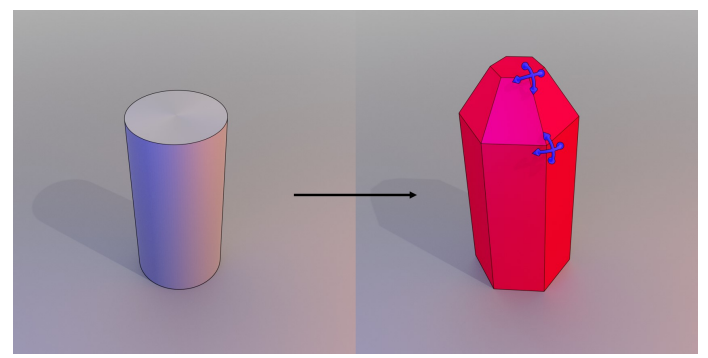

(a)

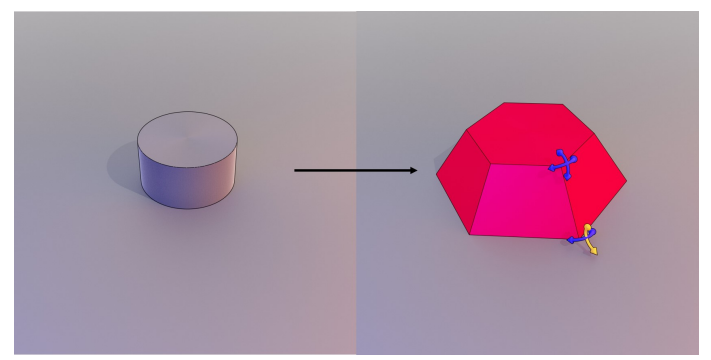

(b)

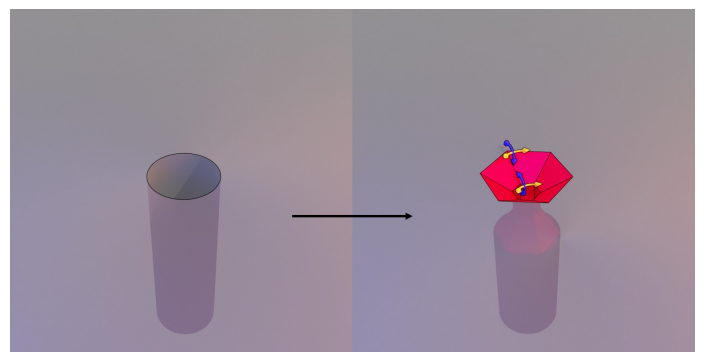

(c)

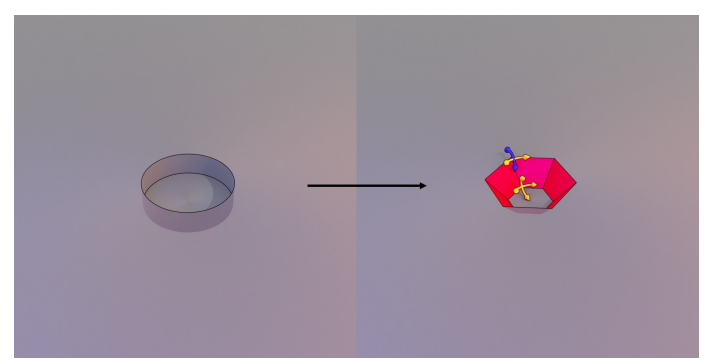

(d)

expand, at the expense of the $c$-plane between the holes.

The shallow hole structures (Figure 6d) have an additional c-plane within the hole upon which growth can occur. The gives a double-concave morphology at the base of the holes causing rapid growth on the semi-polar planes as with the shallow rods. In this case, the top c-plane surrounding the hole structures is slower growing than these semi-polar planes, causing the $c$-plane to expand laterally as growth time increases, reducing the size of the hole structures.

It has been found that the aspect ratio of the holes or rods has a key role in determining whether convex or concave growth occurs and this has a profound impact on the morphology of the structures. This is especially visible after prolonged growth. It also has a knock on effect on whether the top $c$-planes between (holes) or upon (rods) the structures expand or contract.

\subsection{Optimisation of AIN nanostructures for applications}

The introduction described two possible applications for the use of regular arrays of faceted AlN nanostructures: as sites for the creation of quantum dots, and for core-shell UVC emitters. For quantum dots, one requires features on the order of nanometres, commensurate with the excitonic Bohr radius of these materials of $\sim 1.5 \mathrm{~nm}$ [41]. The sharp, highquality, uniform rod apices of the deep rod samples are ideal as sites to house such structures. For core-shell UVC architectures, large, high-quality non- and semi-polar facets for active region growth is desirable to harness the reduced intrinsic QCSE of such devices [35]. Thus a desirable structure would have access to these facets for active region growth and an example of this is a well faceted non-polar rod sidewall.

The small sharp apices of the deep rod samples in Figures 4 (c) and (d) are promising as sites for $\mathrm{GaN}$ or AlGaN quantum dot growth (see figure 8). However, the rods are coalesced which is undesirable as there may be preferential incorporation of material at these coalesced regions. The coalescence also prevents non-polar sidewalls being available for the growth of non-polar quantum well structures for DUV devices. One solution would be to fabricate rods at a larger pitch to avoid coalescence. Another would be to utilise a higher V/III ratio during prolonged growth, and such an experiment is shown in Figure 7.

After prolonged growth (Figure 7 (c) - (e)) the sidewall facets fully meet each other all the way down the rod, since the non-polar $m$-plane growth is considerably slower than that of the $a$-plane. In addition, as the growth time increases (see Figure 7 (e)) the size of the apices becomes very small, beyond the resolution limit of our SEM (see Figure 8). Crucially, even after 270 minutes growth, coalescence between rods has not occurred due to the very slow growing $m$-planes.

In contrast, the deep hole samples are unsuitable for creating sites for quantum dots, for example within the apex of an inverted pyramid, without further optimisation and finetuning, as coalescence is never observed. It appears that the persistent, remaining channel at the centre of the holes is evidence of the self-limited growth nature of these deep etched structures. Furthermore, the shallow hole samples are also not promising due to the irregular bridging and joining within the holes after a prolonged growth time (Figure $4 p)$. However, after optimisation of the dry etch depth of these nanohole structures it may be feasible to achieve fullyformed, well-faceted, inverse pyramid structures. Nevertheless, there is potential for the deep hole and deep rod sam- 
Figure 7: SEM images showing the morphology after different growth durations for the deep nanorod samples at a V/III ratio of 19000. (a) $60 \mathrm{~min}$, (b) $120 \mathrm{~min}$, (c) $180 \mathrm{~min}$, (d) 210 min, (e) $270 \mathrm{~min}$.
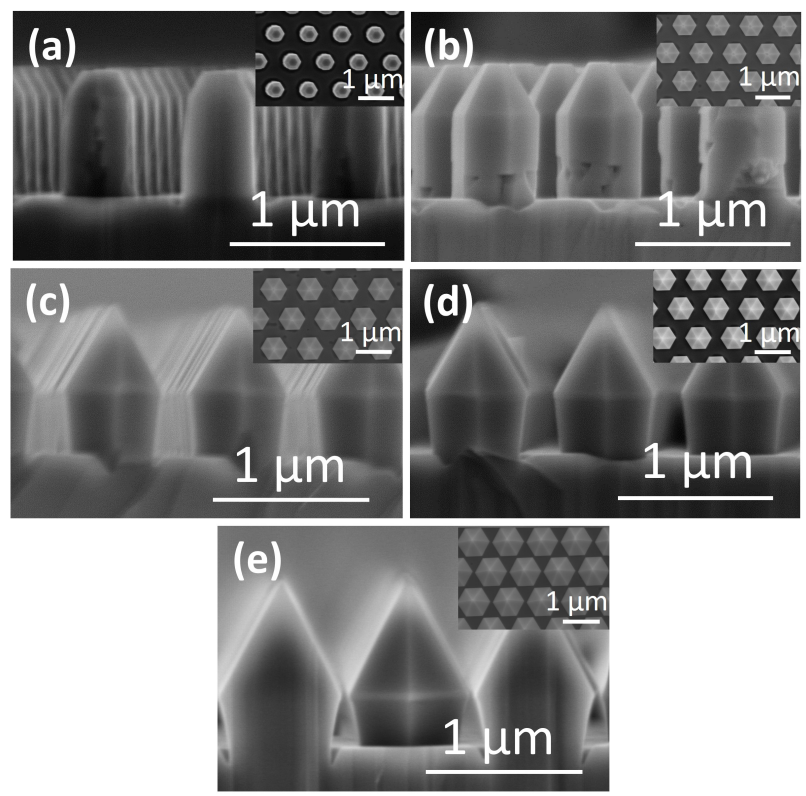

Figure 8: Plot of the size of the top c-plane truncations as a function of rod growth time for the rods grown at V/III $\sim 7600$ and $\sim 19000$. The truncation sizes were extracted from SEM images using ImageJ with estimates of the measurement errors.

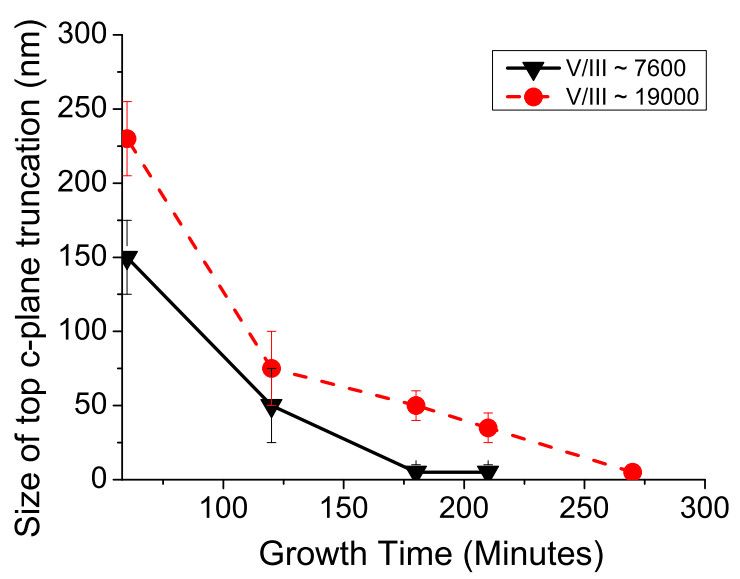

ples to be utilised for the growth of quantum well structures on the non-polar or semi-polar planes for optoelectronic applications in order to take advantage of a reduced quantum confined Stark effect.

\section{Conclusions}

In conclusion, a detailed study of the growth conditions upon the regrowth of AlN nanostructures has been undertaken. The V/III ratio has been found to be more influential than growth temperature on the morphological regrowth of all of the nanostructures investigated. The prolonged growth at a V/III ratio of $\sim 19000$ realised sharp apices at the tips of the nanorods, suitable for the site-control of quantum dots, with the absence of rod coalescence. In addition, well-formed side-walls in the same samples show promise for their potential use in quantum well core-shell structures.

The deep hole samples appear to be self-limited with a hole always remaining at the centre of the structure. Both deep and shallow hole samples start to exhibit mis-oriented, competitive growth from the centre of the structures, demonstrating that it is difficult to form nanopyramidal pit structures without facet competition for a wide range of growth conditions.

Overall, we have shown that the hybrid top-down, bottomup approach has been successful for the realisation of uniform, well faceted AlN nanostructures. This approach shows promise for obtaining previously unattainable devices to be realised with this material.

\section{Experimental Details}

\subsection{Sample Fabrication}

The starting templates were planar, $\sim 4.5 \mu \mathrm{m}$ thick, AlN templates grown via HVPE on a (0001) sapphire substrate which were sourced commercially (Suzhou Nanowin Science and Technology Co. LTD, Suzhou, China). For the creation of nanorods, the AlN wafer was spin-coated with a bottom anti-reflection layer (BARC) (Wide-30W, Brewer Science) and a high-contrast positive resist (Ultra-i 123, Eulitha, Switzerland) with appropriate baking steps. Displacement Talbot lithography (DTL) was used to expose the resist through an amplitude mask (270 $\mathrm{mJ} \mathrm{cm}^{-2}$ exposure dose) with a $1 \mu \mathrm{m}$ pitch. Details about DTL and its applications (e.g. [42]), especially concerning nanopatterning III-N materials can be found elsewhere (see [36]). The BARC and resist were developed (MF-CD-026, Dow Electronic Materials) for a suitable time in order to create an undercut profile in the BARC for subsequent metal dot deposition. Metal dots were deposited through the resist and undercut BARC $(\sim 5$ $\mathrm{nm}$ Au followed by $\sim 210 \mathrm{~nm} \mathrm{Ni}$ ) via e-beam evaporation. Lift-off with developer (MF-CD-26) was performed to reveal a metal dot array to be used as a mask for dry etching. The metal dots had a pitch of $1 \mu \mathrm{m}$ and diameter of $\sim 340$ nm.

For the creation of nanoholes, $\sim 600 \mathrm{~nm} \mathrm{SiN}_{x}$ was deposited onto the AIN template via plasma enhanced chemical vapour deposition (PECVD) (Plasmatherm 790) to be used later as an etch mask. This was followed by spin coating the wafer with BARC (Wide-8C, Brewer Science) and resist (PFI-88, Sumitomo Chemical Advanced Technologies) with appropriate baking steps. DTL was used to expose the resist through an amplitude mask (230 $\mathrm{mJ} \mathrm{cm}^{-2}$ exposure dose) with a $1 \mu \mathrm{m}$ pitch. The wafer was developed (MF-CD-26) with the remaining BARC and resist utilised as a dry etching mask to transfer the pattern through the deposited $\operatorname{SiN}_{x}$. The dry etching into the $\mathrm{SiN}_{x}$ was performed with an inductively 
coupled plasma (ICP) (Oxford Instruments Plasma Technology PlasmaPro 100 Cobra) system with $\mathrm{CHF}_{3}$ gas chemistry at a flow of $25 \mathrm{sccm}$, pressure of $6.5 \mathrm{mTorr}$, RF power $50 \mathrm{~W}$, ICP power $300 \mathrm{~W}$, temperature $20^{\circ} \mathrm{C}$ for 12 minutes. The DC bias was monitored to stay between $270 \mathrm{~V}$ and $266 \mathrm{~V}$. A piranha clean (concentrated $\mathrm{H}_{2} \mathrm{SO}_{4}: \mathrm{H}_{2} \mathrm{O}_{2}$ ) at a ratio of 3:1 was performed for 3 minutes to remove any remaining resist, BARC and any other organic material.

Both sets of samples were cleaned with a gentle $\mathrm{O}_{2}$ based dry etch (via the ICP system stated above) at an RF power $40 \mathrm{~W}$, ICP power $400 \mathrm{~W}$, temperature $20^{\circ} \mathrm{C}$ and pressure of 20 mTorr with an $\mathrm{O}_{2}$ gas flow of $50 \mathrm{sccm}$, to further remove any remaining organic material (such as residual BARC and resist). The metal dot patterned sample was etched for 10 minutes and the DC bias monitored to be between 250-249 $\mathrm{V}$. The $\mathrm{SiN}_{x}$ patterned sample was etched for 5 minutes with the DC bias monitored to be between 250-248 V. A Cl $2 / \mathrm{Ar}$ gas chemistry with flows of $50 \mathrm{sccm}$ and $10 \mathrm{sccm}$ respectively, was then used to etch both samples each to two different etch depths. The RF power was set to $80 \mathrm{~W}$, ICP power $800 \mathrm{~W}$, temperature $150^{\circ} \mathrm{C}$, pressure 14 mTorr, with etch times of 10 minutes and 1 minute. This left four sets of samples, the deep holes and rods, $\sim 1 \mu \mathrm{m}$ in depth, and the shallow holes and rods, $\sim 100 \mathrm{~nm}$ in depth.

An aqua regia clean was then performed on the nanorod samples $\left(\mathrm{HCl}: \mathrm{HNO}_{3}\right.$ ) at a 3:1 ratio for $\sim 5 \mathrm{~min}$ to remove the metal mask. This was followed by a piranha clean (3:1 ratio) for $\sim 3 \mathrm{~min}$ and a buffered oxide etch (BOE) with a 5:1 dilution, for $\sim 2$ min to remove any oxides from the surfaces of the rods. The nanohole samples merely required a BOE at 5:1 dilution for $\sim 50-60 \mathrm{~min}$ for the deep and shallow etched holes to remove both oxides and any remaining $\operatorname{SiN}_{x}$ mask.

\subsection{Regrowth Experiments}

Bottom-up regrowth of AlN was then performed on the samples using a $1 \times 2$ " horizontal MOVPE reactor (AIX 200/4HT RF-S, AIXTRON, Germany). The precursors used were $\mathrm{NH}_{3}$ for nitrogen and trimethlyaluminium for aluminium. The carrier gas during the regrowth was $\mathrm{H}_{2}$. Growth parameters were changed in order to observe the effects on the regrowth of the nanostructures. Specifically, the parameters changed were growth temperature, V/III ratio, and growth time. The reactor chamber pressure throughout all the experiments was kept at 20 mbar.

\subsection{Characterisation}

\subsubsection{Scanning Electron Microscope (SEM)}

The morphological characterisation of the regrown structures and at various stages of the fabrication process were carried out via a scanning electron microscope (SEM) (Hitachi Field-Emission S-4300 SE) using a $5 \mathrm{kV}$ electron beam and detection of the secondary electron signal.

\subsubsection{Atomic Force Microscope (AFM)}

An AFM (Bruker multimode IIIA) was used to analyse the surface facets and macrosteps of the nanohole samples. High resolution silicon AFM tips (supplied from Nunano) with a tip radius of curvature of $<10 \mathrm{~nm}$ were used. Other specifications of the tips include a spring constant of $42 \mathrm{~N} / \mathrm{m}$, resonant frequency of $350 \mathrm{~Hz}$, cantilever dimensions of 125 $\mu \mathrm{m}, 30 \mu \mathrm{m}$ and $4.5 \mu \mathrm{m}$ for the length, width and thickness of the cantilever, respectively. The tips had a high aspect ratio with a cone angle $<15^{\circ}$ over the last $1 \mu \mathrm{m}$. During measurement, the amplitude set point, integral gain and proportional gain were optimised in order to realise high quality images whilst minimising noise. Images were typically taken with a scan time of $1 \mathrm{~Hz}$ per line at a scan size of either 2 or $1 \mu \mathrm{m}$. The data was analysed with the Gwyddion software package.

\section{Acknowledgements}

The authors would like to acknowledge financial support from the EPSRC, UK via Grant Nos. EP/M015181/1 \& EP/M022862/1 "Manufacturing nano-engineered III-nitrides" and from the EPSRC Centre for Doctoral Training in Condensed Matter Physics (CDT-CMP), Grant No. EP/L015544/1. The authors also wish to thank Mr Pierre Chausse (University of Bath, Electronic and Electrical Engineering) for creating the 3D images (Figure 6) as part of the growth mechanism discussion section of this paper. This publication is supported by multiple data sets, which are openly available at https://doi.org/10.15125/BATH-XXXXX.

\section{References}

[1] S. C. Jain, M. Willander, J. Narayan, and R. Van Overstraeten. IIInitrides: Growth, characterization, and properties. Journal of Applied Physics, 87(3):965-1006, 2000.

[2] O. Ambacher. Growth and applications of group III-nitrides. Journal of Physics D: Applied Physics, 31(20):2653-2710, 1998.

[3] S. Strite. GaN, AlN, and InN: A review. Journal of Vacuum Science \& Technology B: Microelectronics and Nanometer Structures, 10(4):1237, 1992.

[4] Michael Kneissl, Tae-Yeon Seong, Jung Han, and Hiroshi Amano. The emergence and prospects of deep-ultraviolet light-emitting diode technologies. Nature Photonics, 13(4):233-244, 2019.

[5] Kazuhito Ban, Jun ichi Yamamoto, Kenichiro Takeda, Kimiyasu Ide, Motoaki Iwaya, Tetsuya Takeuchi, Satoshi Kamiyama, Isamu Akasaki, and Hiroshi Amano. Internal quantum efficiency of wholecomposition-range AlGaN multiquantum wells. Applied Physics Express, 4(5):052101, apr 2011.

[6] S Zhao, AT Connie, MHT Dastjerdi, XH Kong, Q Wang, M Djavid, S Sadaf, XD Liu, I Shih, H Guo, et al. Aluminum nitride nanowire light emitting diodes: Breaking the fundamental bottleneck of deep ultraviolet light sources. Scientific reports, 5:8332, 2015.

[7] Christoph Reich, Martin Guttmann, Martin Feneberg, Tim Wernicke, Frank Mehnke, Christian Kuhn, Jens Rass, Mickael Lapeyrade, Sven Einfeldt, A. Knauer, Viola Kueller, Markus Weyers, Rüdiger Goldhahn, and Michael Kneissl. Strongly transverse-electric-polarized emission from deep ultraviolet algan quantum well light emitting diodes. Applied Physics Letters, 107, 102015.

[8] X. J. Chen, T. J. Yu, H. M. Lu, G. C. Yuan, B. Shen, and G. Y. Zhang. Modulating optical polarization properties of al-rich algan/aln quantum well by controlling wavefunction overlap. Applied Physics Letters, 103(18):181117, 2013.

[9] Dong Yeong Kim, Jun Hyuk Park, Jong Won Lee, Sunyong Hwang, Seung Jae Oh, Jungsub Kim, Cheolsoo Sone, E Fred Schubert, and Jong Kyu Kim. Overcoming the fundamental light-extraction efficiency limitations of deep ultraviolet light-emitting diodes by utilizing transverse-magnetic-dominant emission. Light: Science \& Applications, 4(4):e263, 2015. 
[10] Pierre-Marie Coulon, Gunnar Kusch, Philip Fletcher, Pierre Chausse, Robert W. Martin, and Philip A. Shields. Hybrid top-down/bottomup fabrication of a highly uniform and organized faceted aln nanorod scaffold. Materials, 11(7), 2018.

[11] Michele Conroy, Vitaly Z Zubialevich, Haoning Li, Nikolay Petkov, Sally O'Donoghue, Justin D Holmes, and Peter J Parbrook. Ultrahigh-density arrays of defect-free aln nanorods: A "space-filling" approach. ACS nano, 10(2):1988-1994, 2016.

[12] S Walde, S Hagedorn, P-M Coulon, A Mogilatenko, C Netzel, J Weinrich, N Susilo, E Ziffer, L Matiwe, C Hartmann, et al. Aln overgrowth of nano-pillar-patterned sapphire with different offcut angle by metalorganic vapor phase epitaxy. Journal of Crystal Growth, 531:125343, 2020.

[13] Yu Kee Ooi, Cheng Liu, and Jing Zhang. Analysis of polarizationdependent light extraction and effect of passivation layer for 230$\mathrm{nm}$ algan nanowire light-emitting diodes. IEEE Photonics Journal, 9(4):1-12, 2017.

[14] Jinwan Kim, Uiho Choi, Jaedo Pyeon, Byeongchan So, and Okhyun Nam. Deep-ultraviolet algan/aln core-shell multiple quantum wells on aln nanorods via lithography-free method. Scientific reports, 8(1):935, 2018.

[15] Yanan Guo, Jianchang Yan, Yun Zhang, Junxi Wang, and Jinmin Li. Enhancing the light extraction of AlGaN-based ultraviolet lightemitting diodes in the nanoscale. Journal of Nanophotonics, 12(4):1 $-12,2018$.

[16] Mark J. Holmes, Satoshi Kako, Kihyun Choi, Munetaka Arita, and Yasuhiko Arakawa. Single Photons from a Hot Solid-State Emitter at 350 K. ACS Photonics, 3(4):543-546, 2016.

[17] K. G. Lagoudakis, P. L. McMahon, C. Dory, K. A. Fischer, K. Müller, V. Borish, D. Dalacu, P. J. Poole, M. E. Reimer, V. Zwiller, Y. Yamamoto, and J. Vuckovic. Ultrafast coherent manipulation of trions in site-controlled nanowire quantum dots. Optica, 3(12):1430, 2016.

[18] Jesus Herranz, Lukasz Wewior, Benito Alen, David Fuster, Luisa Gonzalez, and Yolanda Gonzalez. Role of re-growth interface preparation process for spectral line-width reduction of single InAs sitecontrolled quantum dots. Nanotechnology, 26(19), 2015.

[19] Mark J. Holmes, Kihyun Choi, Satoshi Kako, Munetaka Arita, and Yasuhiko Arakawa. Room-temperature triggered single photon emission from a III-nitride site-controlled nanowire quantum dot. Nano Letters, 14(2):982-986, 2014.

[20] Kihyun Choi, Munetaka Arita, Satoshi Kako, and Yasuhiko Arakawa. Site-controlled growth of single GaN quantum dots in nanowires by MOCVD. Journal of Crystal Growth, 370:328-331, 2013.

[21] Chirantan Singha, Sayantani Sen, Pallabi Pramanik, Mainak Palit, Alakananda Das, Abhra Shankar Roy, Susanta Sen, and Anirban Bhattacharyya. Growth of algan alloys under excess group iii conditions: Formation of vertical nanorods. Journal of Crystal Growth, 481:40 - 47, 2018.

[22] L.H. Shen, X.F. Li, J. Zhang, Y.M. Ma, F. Wang, G. Peng, Q.L. Cui, and G.T. Zou. Synthesis of single-crystalline wurtzite aluminum nitride nanowires by direct arc discharge. Applied Physics A, 84(1):7375, Jul 2006.

[23] Qiang Wu, Zheng Hu, Xizhang Wang, Yinong Lu, Kaifu Huo, Shaozhi Deng, Ningsheng Xu, Bo Shen, Rong Zhang, and Yi Chen. Extended vapor-liquid-solid growth and field emission properties of aluminium nitride nanowires. Journal of Materials Chemistry, 13(8):2024-2027, 2003.

[24] Shih-Chen Shi, Surojit Chattopadhyay, Chia-Fu Chen, Kuei-Hsien Chen, and Li-Chyong Chen. Structural evolution of aln nanostructures: Nanotips and nanorods. Chemical Physics Letters, 418(1): 152 - 157, 2006.

[25] V. Cimalla, Ch. Foerster, D. Cengher, K. Tonisch, and O. Ambacher. Growth of aln nanowires by metal organic chemical vapour deposition. physica status solidi (b), 243(7):1476-1480, 2006.

[26] Elena Alexandra Serban, Justinas Palisaitis, Per Ola Åke Persson, Lars Hultman, Jens Birch, and Ching-Lien Hsiao. Site-controlled growth of gan nanorod arrays by magnetron sputter epitaxy. Thin Solid Films, 660:950-955, 2018.
[27] Shota Kitamura, Kazumasa Hiramatsu, and Nobuhiko Sawaki. Fabrication of gan hexagonal pyramids on dot-patterned gan/sapphire substrates via selective metalorganic vapor phase epitaxy. Japanese journal of applied physics, 34(9B):L1184, 1995.

[28] Pierre-Marie Coulon, Blandine Alloing, Virginie Brändli, Denis Lefebvre, Sébastien Chenot, and Jesús Zúñiga-Pérez. Selective area growth of ga-polar gan nanowire arrays by continuous-flow movpe: A systematic study on the effect of growth conditions on the array properties. physica status solidi (b), 252(5):1096-1103, 2015.

[29] Andreas Winden, Martin Mikulics, Toma Stoica, Martina von der Ahe, Gregor Mussler, Anna Haab, Detlev Grützmacher, and Hilde Hardtdegen. Site-controlled growth of indium nitride based nanostructures using metalorganic vapour phase epitaxy. Journal of crystal growth, 370:336-341, 2013.

[30] Mohammed Zeghouane, Geoffrey Avit, Thomas W Cornelius, Damien Salomon, Yamina André, Catherine Bougerol, Thierry Taliercio, Ariane Meguekam-Sado, Pierre Ferret, Dominique Castelluci, et al. Selective growth of ordered hexagonal inn nanorods. CrystEngComm, 21(16):2702-2708, 2019.

[31] Ryan G. Banal, Mitsuru Funato, and Yoichi Kawakami. Surface diffusion during metalorganic vapor phase epitaxy of aln. physica status solidi c, 6(2):599-602, 2009.

[32] George T. Wang, Qiming Li, Jonathan J. Wierer, Daniel D. Koleske, and Jeffrey J. Figiel. Top-down fabrication and characterization of axial and radial iii-nitride nanowire leds. physica status solidi (a), 211(4):748-751, 2014.

[33] Yingdong Tian, Jianchang Yan, Yun Zhang, Yonghui Zhang, Xiang Chen, Yanan Guo, Junxi Wang, and Jinmin Li. Formation and characteristics of algan-based three-dimensional hexagonal nanopyramid semi-polar multiple quantum wells. Nanoscale, 8(21):11012-11018, 2016.

[34] C G Bryce, E D Le Boulbar, P-M Coulon, P R Edwards, I Gîrgel, D W E Allsopp, P A Shields, and R W Martin. Quantum well engineering in InGaN/GaN core-shell nanorod structures. Journal of Physics D: Applied Physics, 50(42):42LT01, sep 2017.

[35] Pierre-Marie Coulon, Gunnar Kusch, Robert W. Martin, and Philip A. Shields. Deep uv emission from highly ordered algan/aln core-shell nanorods. ACS Applied Materials \& Interfaces, 10(39):33441-33449, 2018.

[36] Pierre-Marie Coulon, Benjamin Damilano, Blandine Alloing, Pierre Chausse, Sebastian Walde, Johannes Enslin, Robert Armstrong, Stéphane Vézian, Sylvia Hagedorn, Tim Wernicke, Jesús ZúñigaPérez, Markus Weyers, Michael Kneissl, and Philip Shields. Displacement talbot lithography for nano-engineering of iii-nitride materials. Microsystems \& Nanoengineering, 82019.

[37] Harun H. Solak, Christian Dais, and Francis Clube. Displacement talbot lithography: a new method for high-resolution patterning of large areas. Opt. Express, 19(11):10686-10691, May 2011.

[38] Vibhu Jindal and Fatemeh Shahedipour-Sandvik. Theoretical prediction of GaN nanostructure equilibrium and nonequilibrium shapes. Journal of Applied Physics, 106(8), 2009.

[39] Fangzheng Li, Lianshan Wang, Weizhen Yao, Yulin Meng, Shaoyan Yang, and Zhanguo Wang. Analysis of growth rate and crystal quality of aln epilayers by flow-modulated metal organic chemical vapor deposition. Superlattices and Microstructures, 137:106336, 2020.

[40] Danxu Du, David J. Srolovitz, Michael E. Coltrin, and Christine C. Mitchell. Systematic prediction of kinetically limited crystal growth morphologies. Phys. Rev. Lett., 95:155503, Oct 2005.

[41] Ryota Ishii, Mitsuru Funato, and Yoichi Kawakami. Huge electronhole exchange interaction in aluminum nitride. Phys. Rev. B, 87:161204, Apr 2013.

[42] P. J. P. Chausse, E. D. Le Boulbar, S. D. Lis, and P. A. Shields. Understanding resolution limit of displacement talbot lithography. Opt. Express, 27(5):5918-5930, Mar 2019. 


\title{
Creation of regular arrays of faceted AlN nanostructures via a combined top-down, bottom-up approach Supplementary Information
}

\author{
R. Armstrong, P-M. Coulon, P. Bozinkas, R. Martin and P. Shields \\ ${ }^{1}$ Department of Electronic and Electrical Engineering, University of Bath, Claverton down, Bath, United Kingdom, BA2 7AY \\ ${ }^{2}$ Department of Physics, SUPA, University of Strathclyde, John Anderson Building, 107 Rottenrow, United Kingdom, Glasgow, G4 ONG
}

\begin{abstract}
A B S TR ACT
The realisation of spatially-determined, uniform arrays of faceted aluminium nitride (AlN) nanostructures has had limited exploration, largely due to the fact that selective area growth of AlN via MOVPE (Metal Organic Vapour Phase Epitaxy) has not been realised. Instead, this paper reports the use of a combined top-down, bottom-up approach to realise well-faceted, highly-uniform, periodic nanotextured AlN surfaces. MOVPE regrowth is performed upon dry-etched AlN nanorods and nanoholes, and we present a study into the effect of the growth conditions on the resulting faceting and morphology. Specifically, growth temperature, V/III ratio and growth time are investigated and analysed via scanning-electron and atomic-force microscopy. The V/III ratio was found to influence the nanostructure morphology most whilst the growth temperature was found to have much less of an impact within the temperature range studied. Experiments with a longer growth time are performed to create nanostructures for potential use in applications, such as for AlGaN-based quantum-well or quantum-dot emitters.
\end{abstract}

Figure 9: Calibration image of the AFM data. The black lines indicate measurement lines from which the difference between the know size of $1 \mu \mathrm{m}$ and the measure size is obtained.

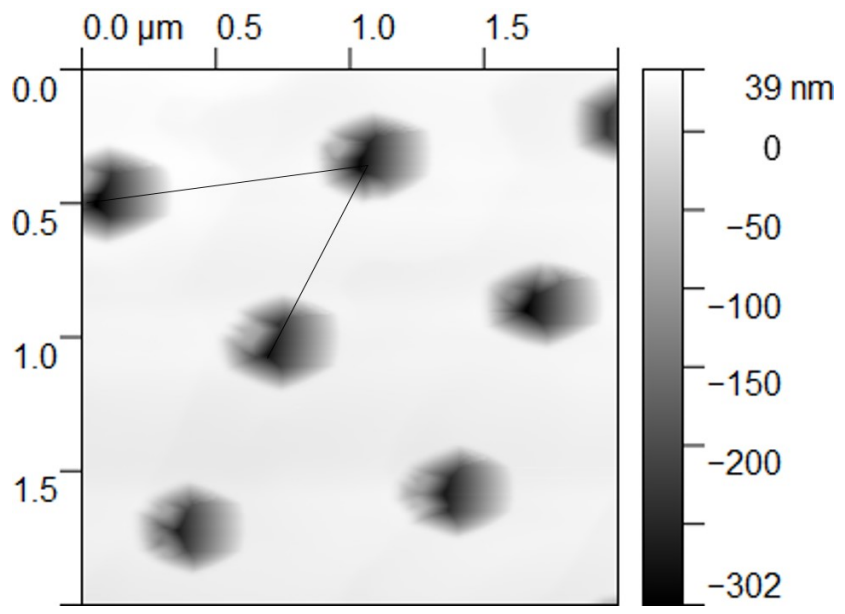

In this supplementary information, extra details and data regarding the AFM measurements mentioned into the main text is provided. All AFM data was analysed with the freely available Gwyddion software package. The AFM raw data of all of the samples was distorted to some extent in terms of the lengths scales of the $\mathrm{x}$ and $\mathrm{y}$ axis measured. All the samples were fabricated with a $1 \mu \mathrm{m}$ pitch; therefore by measuring the pitch of the structures in the $\mathrm{x}$ and $\mathrm{y}$ directions, one could obtain the level of distortion (see black lines in Figure 9). From the obtained dimension measurements the length of the $\mathrm{x}$ and $\mathrm{y}$ axes were re-scaled. This process was per-

\footnotetext{
을 ra760abath.ac.uk (R. Armstrong) $\operatorname{ORCID}(\mathrm{s})$ :
}

formed iteratively until the correct pitch between the structures was obtained. It was often observe that only the ydirection was distorted and not that of the $\mathrm{x}$. This calibration step was performed on scan sizes of either $2 \times 2$ or $5 \times 5 \mu \mathrm{m}$ and then applied to $1 \mathrm{x} 1 \mu \mathrm{m}$ scans under the assumption that the distortion remains constant across all scan sizes. Using this calibration, the pixel ratios of the AFM scans were also adjusted accordingly. From this profile slices, perpendicular to the facets, were taken in order to extract the angles of the semi-polar facets present and thus identify their nature. As all of the samples have edge-to-edge morphology, it is assumed that the semi-polar facets are a-plane in type $\{x x \overline{2 x y}\}$.

The AFM scans of single faceted nanohole structures are shown in figure 10. The angles extracted from the planes are given in table 2. Any misoriented growth observed in the AFM images are neglected and omitted from table 2 as they do not provide a true representation of the semi-polar facets present. The error is estimated to be quite large considering that a reasonable amount of calibratory changes have been made to the AFM scans which has had an effect on the angles extracted comparatively between the raw and adjusted data. Considering that the error is likely to be on the order of $\pm 3-4^{\circ}$ and the samples exhibit edge-to-edge morphology, it is very likely that the semi-polar facets of samples 1 , 2,4 and 6 (in table 2) are that of $\{11 \overline{2} 2\}$. Whilst sample 3 has facet angles similar to that of the other samples, due to the circularity of this sample is is difficult to definitively identify the semi-polar facets present. Indeed, the circularity could mean that a mixture of $\{11 \overline{2} 2\}$ and $\{10 \overline{1} 1\}$ facets are present.

Figure 11 shows the AFM data corresponding to the prolonged growth time study upon the deep hole structures. As 
Figure 10: AFM data of the deep $(a-d)$ and shallow $(e-h)$ holes grown at different V/III ratios.
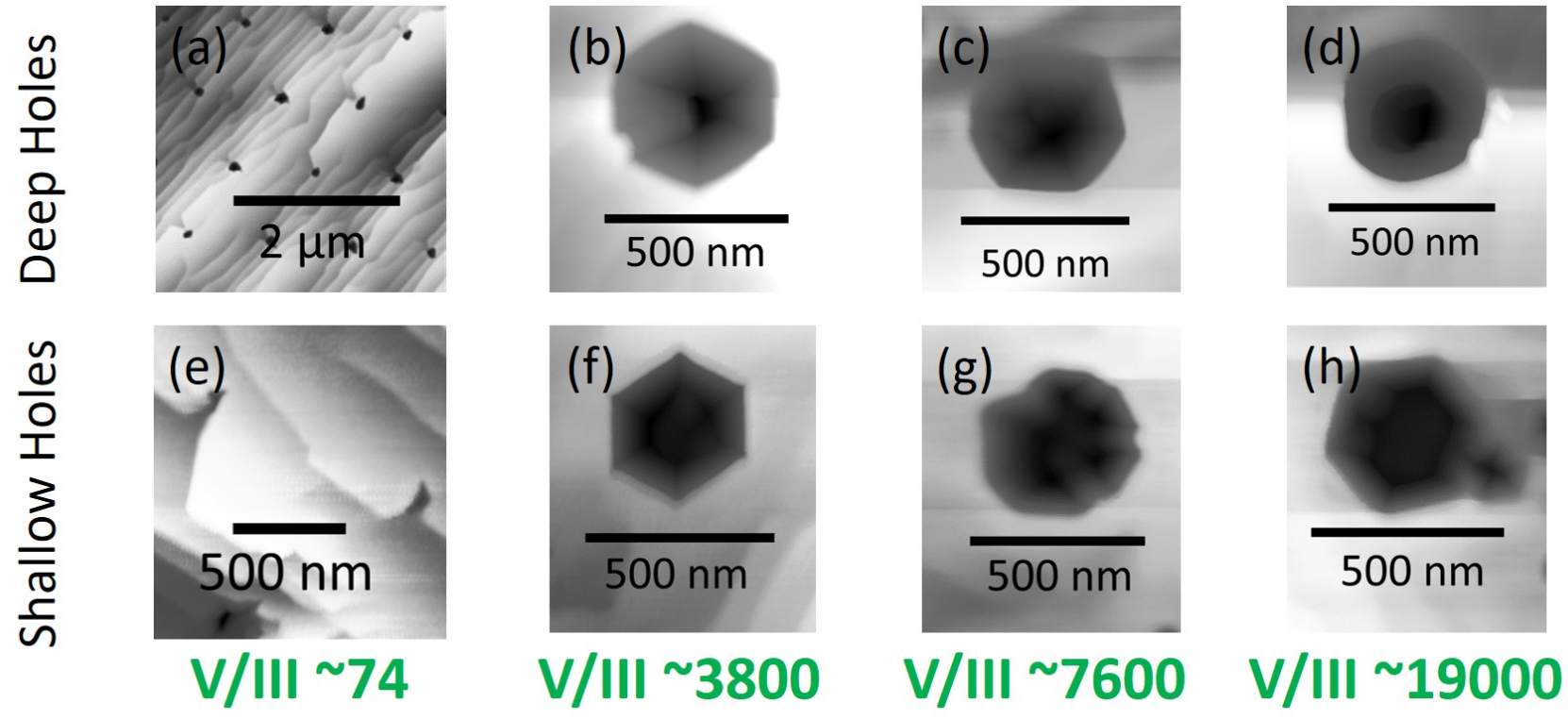

Table 2

This table provides the semi-polar the facet angles measured for the deep and shallow hole structures grown at different $\mathrm{V} / \mathrm{III}$ ratios.

\begin{tabular}{|l|l|l|l|l|}
\hline $\begin{array}{l}\text { Sample } \\
\text { Number }\end{array}$ & Sample & V/III Ratio & Facet Angle Measured & Comments \\
\hline 1 & Deep Holes & $\sim 3800$ & $61-62^{\circ}$ & Some misoriented growth \\
\hline 2 & Deep Holes & $\sim 7600$ & $58-62^{\circ}$ & some misoriented growth \\
\hline 3 & Deep Holes & $\sim 19000$ & $60-62^{\circ}$ & $\begin{array}{l}\text { Circular morphology, likely a lot of mixed, competing } \\
\text { faceting }\end{array}$ \\
\hline 4 & Shallow Holes & $\sim 3800$ & $59-62^{\circ}$ & Some misoriented growth at centre of hole \\
\hline 5 & Shallow Holes & $\sim 7600$ & N/A & $\begin{array}{l}\text { A lot of facet competition, cannot really extract an- } \\
\text { gle date from this sample }\end{array}$ \\
\hline 6 & Shallow Holes & $\sim 19000$ & $58-59^{\circ}$ & Some misoriented growth \\
\hline
\end{tabular}

above, the angles of the semi-polar planes were extracted (see table 3). The angles of samples 1-3 all resided between 59 and $62^{\circ}$. Considering that all the structures have edgeto-edge morphology this data suggests that the semi-polar facets present are again $\{11 \overline{2} 2\}$. Sample 4 of table 3 has a lot of facet competition and misoriented growth present and subsequently the extraction of reliable angles from this structure is not possible. This does however suggest that after a given, prolonged growth time, facet competition arises which is not present in samples grown for a lesser amount of time (comparing Figures 11 (c) and (d)). Thus this data suggests that the observed inverse pyramid with channel structure (Figure 11 (a) -(c)) is not a stable one after prolonged growth. 
Figure 11: AFM data of the growth time study of the deep holes at a V/III ratio of $\sim 7600$.

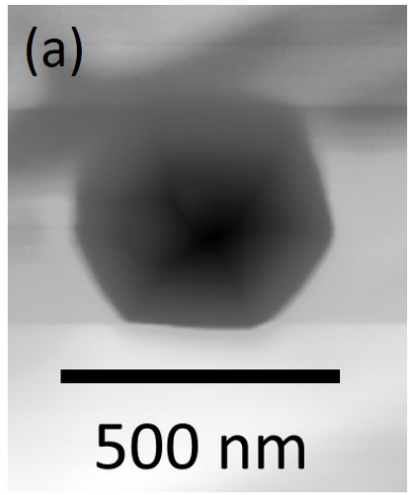

60

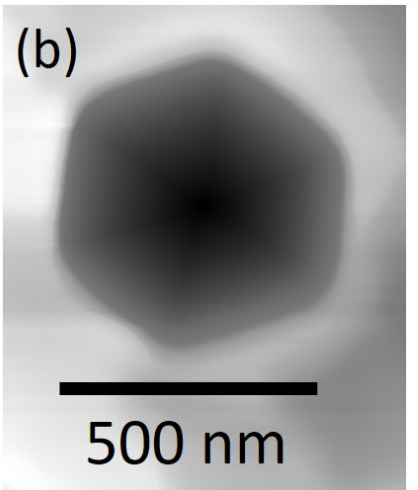

120

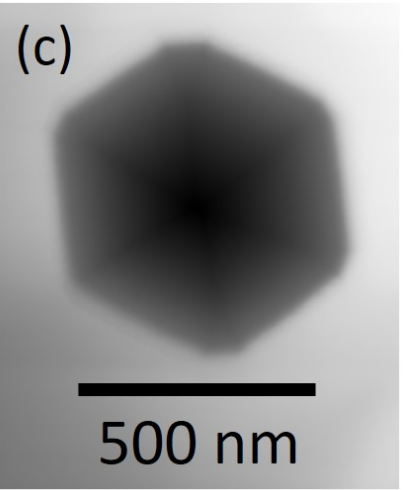

150 (d)

\section{$500 \mathrm{~nm}$}

\section{Growth time (minutes)}

Table 3

This table provides the semi-polar the facet angles measured for the deep hole structures grown at a V/III ratio of $\sim 7600$ for prolonged growth times.

\begin{tabular}{|l|l|l|l|}
\hline $\begin{array}{l}\text { Sample } \\
\text { Number }\end{array}$ & $\begin{array}{l}\text { Growth } \\
\text { Time } \\
\text { (Minutes) }\end{array}$ & Facet Angle Measured & Comments \\
\hline 1 & 60 & $61-62^{\circ}$ & Some misoriented growth \\
\hline 2 & 120 & $59-61^{\circ}$ & Well faceted structure \\
\hline 3 & 150 & $60-61^{\circ}$ & Well faceted structure \\
\hline 4 & 180 & N/A & A lot of misoriented growth and facet competition \\
\hline
\end{tabular}

